Mh. Math. 125, 189-209 (1998)

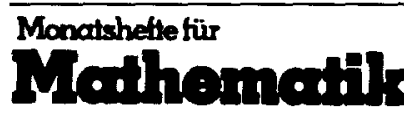

CSpringer-Verlag 1998

Printed in Austria

\title{
Closed Curves and Geodesics with Two Self-Intersections on the Punctured Torus
}

\author{
By \\ David Crisp, Adelaide, Susan Dziadosz, Ann Arbor, MI, Dennis J. Garity, \\ Corvallis, OR, Thomas Insel, Berkeley, CA, Thomas A. Schmidt, Corvallis, CA, \\ and Peter Wiles, Madison, WI
}

With 12 Figures

(Received 11 March 1996; in revised form 15 January 1997)

\begin{abstract}
We classify the free homotopy classes of closed curves with minimal self intersection number two on a once punctured torus, $T$, up to homeomorphism. Of these, there are six primitive classes and two imprimitive. The classification leads to the topological result that, up to homeomorphism, there is a unique curve in each class realizing the minimum self intersection number. The classification yields a complete classification of geodesics on hyperbolic $T$ which have self intersection number two. We also derive new results on the Markoff spectrum of diophantine approximation; in particular, exactly three of the imprimitive classes correspond to families of Markoff values below Hall's ray.
\end{abstract}

\section{Introduction}

We classify the free homotopy classes of loops on a once-punctured torus, $T$, whose self-intersection number is two. Our classification is up to homeomorphism type; that is, we identify two free homotopy classes if there is a self homeomorphism of the punctured torus taking one class to the other. There are eight such classes. This classification has topological, geometric and number theoretic consequences.

The classification leads to the topological result that each homeomorphism class of free homotopy classes of intersection number two contains a unique curve, up to homeomorphism, realizing the minimum intersection number. In addition, all but two of the classes are distinguished by the closures of the complementary domains of any curve in the class that realizes the minimum self intersection number. In particular, if two curves have self intersection number two and are not freely homotopic to curves with lower self intersection number, and if they are in the same class, then there is a homeomorphism of $T$ taking one curve to the other.

A geometric consequence of the classification is that the classes we obtain include the classes which, for any hyperbolic metric, admit a closed geodesic of

1991 Mathematics Subject Classification 53A35, 57M50; 11 J06

Key words: geodesic, punctured torus, Markoff spectrum, diophantine approximation

Research started during the Summer 1994 NSF REU Program at Oregon State University and partially supported by NSF DMS 9300281 
two self-intersections. Thus, given a hyperbolic metric on $T$, we obtain a classification of geodesics on $T$ with two self intersections. Up to homeomorphism of $T$, and thus up to automorphism of the fundamental group of $T$, there are six such classes.

In work which responds to C. SERIES' closing question in [15], D. CRISP and W. MORAN [6], [5], [7] classified the singly self-intersecting geodesics of a particular hyperbolic punctured torus; they then proceeded to obtain results about the Markoff spectrum of diophantine approximation. We use our classification of doubly self-intersecting geodesics on $T$ to obtain further information about the Markoff spectrum.

The simple curves on a punctured torus are well studied: for the use of studying the generators of the fundamental group and the action of its automorphisms [11], [3]; for identifying Teichmüller space [13], [18]; and for their relationship to the Markoff spectrum of diophantine approximation [4] (for an overview), [9], [16]. That the geodesics of a hyperbolic punctured torus are rather special is well known, see [19] for a convincing theorem to this effect. That these geodesics are somehow fundamental in the study of more general surfaces is demonstrated in [2; Chapter 3].

We also point out here that classifications of the nature we obtain are actually valid for all hyperbolic metrics on the punctured torus. We mention that our method easily generalizes to the case of higher self-intersection numbers. Although dependent upon earlier works, our approach is somewhat new.

This paper is an outgrowth of an NSF REU project at Oregon State University. Crisp and Schmidt thank Bill Moran for earlier related conversations.

In Section 1, we give the topological background for this project and in Section 2, we give the geometric and number theoretic background. In Section 3, we obtain the actual classification. The case analysis involved uses standard combinatorial topological methods. The proof that the classes obtained are distinct is more interesting and is postponed until a later section.

In Section 4, we give the topological consequences of the classification, in Section 5 we give the geometric consequences, in Section 6 we prove the distinctness of the classes and in Section 7 we discuss the Markoff values associated to the geodesics for a particular hyperbolic metric. We close with some questions and suggestions for further research.

\section{Topological Background}

Let $T$ be a punctured torus. The fundamental group of $T, \pi_{1}(T)$, is isomorphic to the free group on two letters, $F(a, b)$. We fix such an isomorphism. There is a natural bijection between free homotopy classes of closed curves on $T$ and conjugacy classes of elements of $F(a, b)$.

A loop is said to have a single nontrivial self-intersection if the loop has a single transverse intersection, and is not freely homotopic to a simple loop. A loop is said to have two nontrivial self-intersections if it has two transverse selfintersection points, and if it is not freely homotopic to a loop with a single nontrivial self-intersection or to a simple loop. A free homotopy class of loops is 
said to have intersection number two if it contains a loop with two nontrivial selfintersections.

Our initial goal is to classify those free homotopy classes with intersection number two. The classification will be as follows. We view a loop as a map from $S^{1}$ into $T$. Given a free homotopy class which contains a loop $\ell$ with two nontrivial intersections, we show that the class is completely determined by the pair $\left(T, \ell\left(S^{1}\right)\right)$. That is, if $\ell^{\prime}$ is another loop with two nontrivial self intersections in the class, then there is a homeomorphism $h$ of pairs taking $\left(T, \ell\left(S^{1}\right)\right)$ to $\left(T, \ell^{\prime}\left(S^{1}\right)\right)$. Moreover, this homeomorphism can be chosen so as to preserve orientation in the sense that there is an orientation preserving homeomorphism $k$ of $S^{1}$ so that $h \circ \ell=\ell^{\prime} \circ k$.

We also show that a free homotopy class of intersection number two is completely determined by the pattern of closures of the complements of a loop $\ell$ realizing the minimum intersection number.

We state a result that follows directly from the classification of surfaces. See BIRMAN and SERIES [1] for a discussion of this and other results about simple loops on surfaces.

Theorem 1.1. The conjugacy class of a simple loop $l$ on $T$ is either

(a) the identity, and l bounds a disc,

(b) one of $\left[a b a^{-1} b^{-1}\right]$ or $\left[b a b^{-1} a^{-1}\right]$, and l bounds punctured disc, or

(c) $[w]$ where $w$ is a generator of $\pi_{1}(T)$, and $l$ does not separate $T$.

In [6], [5] and [7], a classification of loops with a single nontrivial selfintersection is given. The methods used are to apply the above result to each of the subloops.

Theorem 1.2. (Crisp). The conjugacy class in $\pi_{1}(T)$ of a loop with a single nontrivial self-intersection on $T$ is one of

(a) $\left[\left(a b a^{-1} b^{-1}\right)^{2}\right]$ or $\left[\left(a b a^{-1} b^{-1}\right)^{-2}\right]$,

(b) $\left[g\left(a^{2}\right)\right]$ for some $g \in$ Aut $\pi_{1}(T)$,

(c) $\left[g\left(a b a b^{-1}\right)\right]$ for some $g \in$ Aut $\pi_{1}(T)$, or

(b) $\left[g\left(a a b a^{-1} b^{-1}\right)\right]$ for some $g \in$ Aut $\pi_{1}(T)$.

Conversely, each of these conjugacy classes contains such a loop.

\section{Geometric Background}

We now let $T$ be a punctured torus with a hyperbolic metric. A free homotopy class of closed curves on $T$ is said to be primitive if it is not a non-trivial power of some other class.

It is well known that a closed curve on $T$ is freely homotopic to a geodesic if and only if the curve lies in a primitive free homotopy class which contains no simple curve bounding either a disc or a punctured disc. Furthermore, a geodesic has the minimal number of self-intersections amongst all curves in its free homotopy class.

J. NIELSEN [11] showed that every automorphism of $\pi_{1}(T)$ can be realized, up to inner automorphism, by some homeomorphism. He further showed that every 
automorphism of $\pi_{1}(T) \cong F(a, b)$ takes $a b a^{-1} b^{-1}$ to $x\left(a b a^{-1} b^{-1}\right)^{ \pm 1} x^{-1}$ for some $x \in F(a, b)$.

Theorem 2.1. Let $T_{1}$ and $T_{2}$ be two hyperbolic punctured tori. Let $\left(A_{1}, B_{1}\right)$ and $\left(A_{2}, B_{2}\right)$ be fixed generating pairs of their respective fundamental groups. Let $n$ be a positive integer. Let $W(a, b)$ be $a$ word in the letters $a$ and $b$. If a geodesic on $T_{1}$ in the free homotopy class of $W\left(A_{1}, B_{1}\right)$ has $n$ intersections, then any geodesic on $T_{2}$ in the free homotopy class of $W\left(A_{2}, B_{2}\right)$ also has $n$ intersections.

Proof. There is a homeomorphism between $T_{1}$ and $T_{2}$, call it $h$. The images of the free homotopy classes $\left[A_{1}\right]$ and $\left[B_{1}\right]$ are, say, $[\alpha]$ and $[\beta]$. Similarly, $\left[W\left(A_{1}, B_{1}\right)\right]$ is sent to $[W(\alpha, \beta)]$. Since $h$ is a homeomorphism, these have the same minimal number of self-intersections.

Since $h$ induces an isomorphism on $\pi_{1}, \alpha$ and $\beta$ are a generating pair for $\pi_{1}\left(T_{2}\right)$. In particular, there is an automorphism of $\pi_{1}\left(T_{2}\right)$ taking $(\alpha, \beta)$ to $\left(A_{2}, B_{2}\right)$. This automorphism sends $[W(\alpha, \beta)]$ to $\left[W\left(A_{2}, B_{2}\right)\right]$. But, the automorphism is realizable by a homeomorphism, hence the minimal number of self-intersections in these classes must be the same. Since the geodesic in each of these classes achieves this minimal number of self-intersections, we are done.

Corollary 2.2. A classification for a particular hyperbolic punctured torus of the automorphism classes of those free homotopy classes which contain closed geodesics which are n-times self-intersecting is in fact valid for all such punctured tori.

Remark. We have thus shown that the results of [6], as proven in [5] and [7], are in fact valid for all hyperbolic punctured tori. Indeed, the classification is fundamentally topological. We point out that it has consequences even for arbitrary Riemannian metrics on the punctured torus: a class which for a hyperbolic metric has a unique closed geodesic with $m$ self-intersections, will for an arbitrary Riemannian metric admit a geodesic of $m$ self-intersections; such a class may admit geodesics with more self-intersections, but never less. Thus, a classification of the classes which for a hyperbolic metric admit a geodesic with up to $m$ self-intersections identifies the set of classes which for an arbitrary Riemannian metric may admit geodesics of $m$ self-intersections.

For the classification of twice self-intersecting geodesics, we specialize to a fixed metric only as one means for showing that two homeomorphism classes of geodesics are distinct. The surface we then use is the quotient of the Poincare upper half-plane by the commutator subgroup of the modular group, exactly that studied by the above mentioned authors. Indeed, we will also give number theoretic implications of our classification by interpreting them with respect to this particular metric.

\subsection{Specialization to the $\Gamma^{\prime}$-Metric}

We consider the particular once-punctured torus $\mathbf{T}$, the quotient of the Poincare upper half-plane, $\mathscr{H}$, by the commutator subgroup of the modular group, $\Gamma^{\prime}$. This torus has constant curvature minus one - thus, there is at most one geodesic in 
each free homotopy class - and admits $\mathscr{H}$ as its universal Riemannian cover. The action of $\Gamma$ on $\mathscr{H}$ is given by linear fractional transformations. We use a standard fundamental domain $\mathscr{D}$ for this action - a quadrilateral with hyperbolic line segment boundaries of vertices $-1,0,1, \infty$.

$$
\text { We take } A=\left(\begin{array}{ll}
1 & 1 \\
1 & 2
\end{array}\right) \text { and } B=\left(\begin{array}{cc}
1 & -1 \\
-1 & 2
\end{array}\right) \text { as generators of } \Gamma^{\prime} \cong F(A, B) \text {. }
$$

Thus, given a word $W$ in $A$ and $B$, by matrix multiplication we find a corresponding matrix in $\Gamma^{\prime}$. A hyperbolic element of $\Gamma^{\prime}$ is one of trace greater than 2 in absolute value. Each such fixes an axis - a geodesic of $\mathscr{H}$, thus a semi-circle with center on $\mathbb{R} \cup\{\infty\}$. Free homotopy classes correspond to conjugacy classes in $\Gamma^{\prime}$. A free homotopy class has a closed geodesic in it if and only if the corresponding conjugacy class is hyperbolic. The geodesic in such a homotopy class is then the projection of the axes fixed by the elements of the conjugacy class. Indeed, the geodesic is the 1-1 projection of those segments of the fixed axes which lie in $\mathscr{D}$, where we note that $\mathscr{D}$ has certain sides paired by the actions of $A$ and $\mathrm{B}$. Furthermore, for a reduced word in $A$ and $B$ representing an element of the conjugacy class, the cyclic permutations of this word determine all axes which will have geodesic segments within $\mathscr{D}$. We refer to [5] for a more detailed presentation of these standard facts.

Thus, to find the number of self-intersections of the unique geodesic in the class [W], for a given (hyperbolic) word $W$ in $A$ and $B$, we find the number of intersections within $\mathscr{D}$ of the axes of those matrices which arise from the cyclic permutations of the word of $W$. That is, for each cyclic permutation of $W$ we find the corresponding element $M \in \Gamma^{t}$ as a matrix. There is a straightforward formula for the endpoints $p_{1}, p_{2} \in \mathbb{R}$ of the axis of $M$. Thus, we find each axis. We then simply count the number of intersections which lie in $\mathscr{D}$ of these finitely many axes. Note that this determination of the number of self-intersections of the geodesic of $W$ has thus been reduced to arithmetic.

Thus, by Theorem 2.1, we can determine the self-intersection number of any free homotopy class for a general once-punctured torus. In what follows we will refer to the result of such a calculation as coming from specialization to the $\Gamma^{\prime}-$ metric.

\subsection{Geodesics Mapped to Geodesics}

We will have need to decide whether a specific pair of free homotopy classes are equivalent up to the action of the outer automorphisms of the fundamental group. As we will discuss in Section 5, there are various ways to address this problem. One is to specialize to a particular hyperbolic metric and to consider the action of the Teichmüller modular group.

As discussed in say [10], the Teichmüller modular orbit of a hyperbolic punctured torus arises from a relabeling of generators of the fundamental group. That is, the action of the outer automorphisms of the fundamental group are realized by that of the Teichmüller modular group. Moreover, within an orbit, this action can be given by way of isometries of the universal Riemannian cover, $\mathscr{H}$. In particular, the action of the automorphisms can be realized by diffeomorphisms of hyperbolic punctured tori which send geodesics to geodesics. 
Thus, for geodesics with respect to a fixed hyperbolic metric to be related under some outer automorphism, the complements of the geodesics must be of the same topological type. This is a true strengthening over the mere assumption that outer automorphisms be realized by homeomorphisms - under this, we merely know that a geodesic in a class is sent to some curve in the class of the second; free homotopy could allow for vastly different complements.

This brief discussion thus indicates that two free homotopy classes are not equivalent under the action of the outer automorphisms of the fundamental group if under some specialization to a hyperbolic metric the complements of their respective geodesics have differing topological type. We will use this in Section 5 .

\subsection{Geometry of the Markoff Spectrum}

In the case of the $\Gamma^{\prime}$-metric, the simple closed geodesics are known to be in 1-1 correspondence with the Markoff numbers of Diophantine approximation. Briefly, the Markoff spectrum can be considered as the set of suprema of Euclidean diameters of lifts of the geodesics of $\Gamma^{\prime} \backslash \mathscr{H}$. It is known that there is a ray approaching infinity, the Hall ray, of values in this spectrum; for further details, see the discussion of [8]. A. HAAS [9] remarked that any geodesic which has a loop about the puncture will have Markoff value at least 6 . But, 6 is known to be in the Hall ray of the Markoff spectrum. The lowest part of the spectrum corresponds to the simple closed geodesics. The intermediate section of the Markoff spectrum is still rather mysterious.

Another characterization of the Markoff spectrum is in terms of continued fractions. Recall that a real number can be expanded in a continued fraction of the form

$$
a_{0}+\frac{1}{a_{1}+\frac{1}{\ddots}},
$$

where $a_{0}$ is an integer and the remaining $a_{i}$ are positive integers. We use a flat notation for this continued fraction expansion: $\left[a_{0} ; a_{1}, \ldots\right]$. Given a doubly infinite sequence of positive integers, $\mathscr{A}=\ldots, a_{-1}, a_{0}, a_{1}, \ldots$, one defines $\lambda_{i}(\mathscr{A})$ to be $\left[a_{i} ; a_{i+1}, \ldots\right]+\left[0 ; a_{i-1}, a_{i-2}, \ldots\right]$. The Markoff value of $\mathscr{A}$ is the supremum of the $\lambda_{i}(\mathscr{A})$. The above mentioned Markoff numbers is the discrete set of values with unique limit value 3 , given by certain, well-characterized, periodic doubly infinite sequences of 1, 1's and 2, 2's. For further details, we again refer to [8].

The above indicates that the Markoff values associated to the simple closed geodesics for the $\Gamma^{\prime}$-metric are isolated within the Markoff spectrum. The CrispMoran conjecture, introduced in [6], is that all Markoff values corresponding to the single self intersecting geodesics which contain no loop about the puncture are also isolated within the Markoff spectrum. Thus, there are important aspects of the Markoff values associated to geodesics of the $\Gamma^{\prime}$-metric which are known to depend only upon the homeomorphism class of the geodesics. This is what in part motivated the present work. 


\section{Loops with Two Self-Intersections}

Our goal in this section is to classify loops on $T$ with two nontrivial selfintersections. We consider the composition of three simple loops, as justified by the following lemma, obtained by the authors Dziadosz, Insel, and Wiles.

Lemma 3.1. Up to free homotopy, any loop $l$ on $T$ with $k$ transverse selfintersection points can be formed as the composition of $k+1$ simple loops, which intersect at only one point.

Proof. Consider a loop $l$ on $T$ with $k$ transverse self-intersections occurring at distinct points. Call a segment that part of the loop which is minimally between two (possibly nondistinct) points of intersection. We claim that there are $2 k$ distinct segments connecting the intersections of $l$. Indeed, as four (possibly nondistinct) segments converge at each intersection and each segment connects two intersections, there are exactly $4 k / 2=2 k$ total distinct segments connecting the self-intersections of $l$.

Now, pick some point $p$ on $T$. For each self-intersection of $l$, we can continuously deform $l$ so that the intersection occurs at $p$, in effect collapsing a segment of $l$. In order to bring all intersections to one point, we perform $k-1$ collapsings, leaving $2 k-(k-1)=k+1$ segments.

The lemma shows that it is sufficient to consider the compositions of three simple loops in order to determine all loops with two transverse self-intersections. In addition, the following Corollary to this Lemma limits the number of cases that need to be considered. This Corollary follows immediately from the manner in which the three simple loops were obtained in the Lemma.

Corollary 3.2. Let l be a loop on T formed as the composition of 3 simple loops which intersect only at a single point $p$. Assume that $l$ arises as in the Lemma from a loop $\tilde{l}$ with 2 transverse intersections. Then the point $p$ has a neighborhood $U$ in $T$ such that $U \cap l$ is a wedge of six segments with wedge point $p$ as in Figure 1. Moreover, this wedge has a unique "axis" c-d as in Figure 1 along which the collapsing was done to obtain $l$ from $\tilde{l}$, and $\tilde{l}$ can be recovered from $l$ by reversing the collapsing along the axis $c-d$.

Corollary 3.3. Let $l$ and $U$ be as in Corollary 3.2. Suppose that $l$ is the composition of loops $l_{1}, l_{2}$ and $l_{3}$ of basepoint $p$ of $T$. For $i \in\{1,2,3\}$, let $i$ and $i^{\prime}$ label respectively the initial and final segments of $l_{i} \cap U$. The resulting graph, which we call a basepoint graph, is one of the graphs in Figure 2.

Proof. We use a cyclic ordering on $\{1,2,3\}$. From the graph of $l \cap U$, labelling some segment as $i^{\prime}$ also determines the segment labelled $i+1$. Moreover, the axis
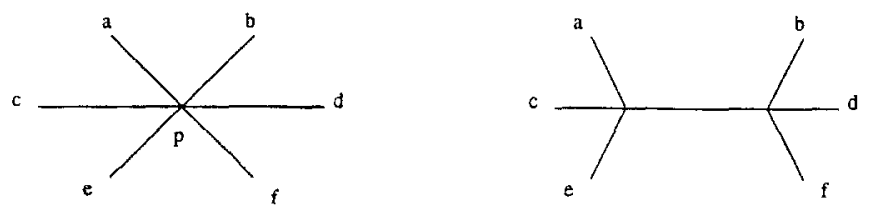

Figure 1. Basic wedge pattern 
A

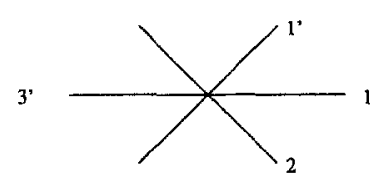

$(1,2,, 3 ;,, 1 ;)$

D

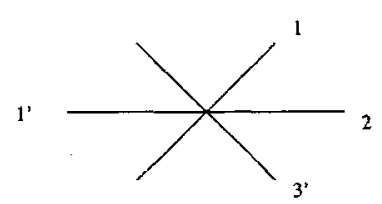

$\left(1,2,3,, 1,1_{-}\right)$
B

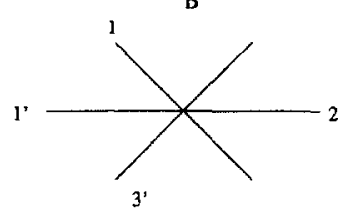

$\left(1, \ldots, 2,3^{*}, 1\right)$

E

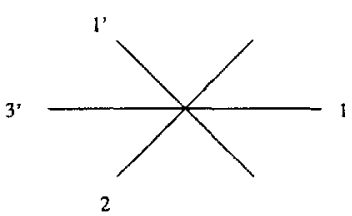

$\left(1, \ldots, 2,3^{\prime}, 1^{\prime}, \ldots\right)$

G

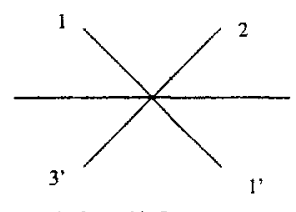

$\left(1,2, \ldots, 1^{\prime}, 3, \ldots\right)$

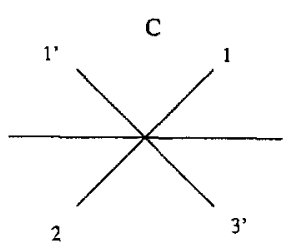

$\left(1, \ldots, 3,2, \ldots, 1^{\prime}\right)$

\section{F}

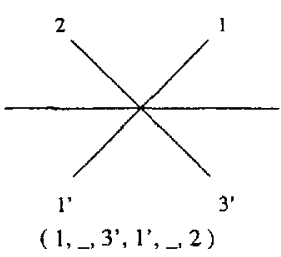

Figure 2. Possible basepoint graphs

must be labelled $\left(j^{\prime}, j+1\right)$ for some $j$. One now lists all possible basepoint graphs. We label clockwise, beginning with 1 , and consider the number of segments between $1^{\prime}$ and $1: 1^{\prime}$ and 1 are adjacent $(\mathrm{A}, \mathrm{B}, \mathrm{C})$; separated by one segment (D, $\mathrm{E})$; by two (F, G). Note that in each of these cases there are two unlabeled segments, allowing for a choice of orientation.

We will refer to an analysis of basepoint segment pattern as the process of determining which $\{A, \ldots, G\}$ could come from a given configuration of (often unoriented) loops $l_{i}$. In practice, this will allow us to determine which orientations of the $l_{i}$ are possible. In general, this process will be in two steps. Since the $l_{i}$ are (in general) initially known only up to orientation, we first consider $j^{\prime}$ as equivalent to $j$ and write out the possible sequences of $j \in\{1,2,3\}$ coming from the pattern of the $l_{i}$. Thus, each $j$ appears twice in each such sequence. This is followed by determining the possible ways to label one of each pair of $j$ with a prime so as to achieve one of the basepoint graphs in $\{A, \ldots, G\}$.

We continue now by classifying the possible compositions of three simple loops that can arise from loops with two transverse intersections.

Theorem 3.4. Let $l$ be a loop on $T$ formed as the composition of 3 simple loops which intersect only at a single point. Assume that l arises as in Lemma 3.1 from a 
loop $\tilde{l}$ with precisely 2 transverse intersections. Then the conjugacy class of $l$ in $\pi_{1}(T),[l]$, is one of
(a) $\left[g\left(a a b a^{-1} b^{-1} a b a^{-1} b^{-1}\right)\right]$,
(b) $\left[g\left(a a a b a^{-1} b^{-1}\right)\right]$,
(c) $[g(a a a b b)]$,
(d) $\left[g\left(a b a b^{-1} a b a^{-1} b^{-1}\right)\right]$,
(e) $\left[g\left(a a b a^{-1} a^{-1} b^{-1}\right)\right]$,
(f) $\left[g\left(a a b a b^{-1}\right)\right]$,
(g) $\left[g\left(a^{3}\right)\right]$,
(h) $\left[g\left(\left(a b a^{-1} b^{-1}\right)^{3}\right)\right]$,
(i) the conjugacy class of a loop with one non-trivial self-intersection, or
(j) the conjugacy class of a simple loop for some $g \in$ Aut $\pi_{1}(T)$.

Proof. Let $l=l_{1} l_{2} l_{3}$, where $l_{1}, l_{2}$ and $l_{3}$ are simple loops on $T$. We can move the common intersection point of the simple loops to the basepoint of $\pi_{1}(T)$ by an isotopy of $T$. Let $w, w_{1}, w_{2}$ and $w_{3}$ be the homotopy classes of $l, l_{1}, l_{2}$ and $l_{3}$ respectively. The corresponding free homotopy classes are $[w],\left[w_{1}\right],\left[w_{2}\right]$ and $\left[w_{3}\right]$.

Note that $[w]=\left[w_{1} w_{2} w_{3}\right]=\left[w_{3} w_{1} w_{2}\right]=\left[w_{2} w_{3} w_{1}\right]$ since cyclic permutations are conjugate by the obvious elements of the free homotopy group.

In the following proof, we use without explicit mention the topology of simple curves on the torus. RoLFSEN's book on knot theory contains the necessary results [12]. Often, after cutting apart the torus along a non-separating curve, we work with simple curves on the resulting punctured disc. The possibilities that arise for the various curves come from repeated application of the Schoenflies theorem.

By Theorem 1.1, each of $l_{1}, l_{2}$ and $l_{3}$ can be either the identity, a generator, or a loop around the puncture. Thus, the following cases arise:

Case 1 . For some $i,\left[w_{i}\right]=[\mathrm{Id}]$;

Case 2. Each of $\left[w_{1}\right],\left[w_{2}\right],\left[w_{3}\right] \in\left\{\left[a b a^{-1} b^{-1}\right],\left[b a b^{-1} a^{-1}\right]\right\}$;

Case 3. For some $i,\left[w_{i}\right]=[g(a)]$ and $\left\{\left[w_{j}\right] \mid j \neq i\right\} \subset\left\{\left[a b a^{-1} b^{-1}\right],\left[b a b^{-1} a^{-1}\right]\right\}$;

Case 4. Exactly two of $\left[w_{1}\right],\left[w_{2}\right],\left[w_{3}\right]$ are of the form $[g(a)]$ for some automorphism $g$ of $\pi_{1}(T)$, and the third is in $\left\{\left[a b a^{-1} b^{-1}\right],\left[b a b^{-1} a^{-1}\right]\right\}$; and

Case 5. All of the homotopy classes represent generators.

Since the details involved in the above cases are similar, we provide a complete argument only for Case 1, Case 2 and a few subcases of Case 4 .

Case 1: Some $\left[w_{i}\right]=[\mathrm{Id}]$.

Without loss of generality, $i=1$. In this case, $[w]=\left[w_{2} w_{3}\right]$, the conjugacy class of either a simple loop or a loop with one non-trivial self-intersection. In fact, Corollary 3.2 shows that if we recover the loop $\tilde{l}$ with two nontrivial intersections from $l$, then the part of $\tilde{l}$ corresponding to $l_{1}$ is as pictured in Figure 3 where the region enclosed by the curve is a disc on $T$. In the first case in this figure, one intersection can be removed by a free homotopy and the loop is freely homotopic
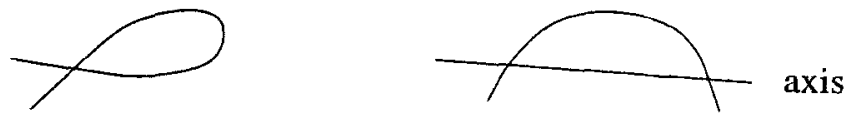

Figure 3. Figure for Case 1 
to a loop with a single transverse self intersection. In the second case in this figure, both intersections can be removed by a free homotopy and the loop is freely homotopic to a simple loop.

Case 2: $\left[w_{1}\right],\left[w_{2}\right],\left[w_{3}\right] \in\left\{\left[a b a^{-1} b^{-1}\right],\left[b a b^{-1} a^{-1}\right]\right\}$.

Each of the loops $l_{i}$ is a loop bounding a punctured disc. We know that $\left[a b a^{-1} b^{-1}\right]$ and $\left[b a b^{-1} a^{-1}\right]$ are the free homotopy classes of simple loops around the puncture, and that they have opposite orientations. Without loss of generality, $l_{1}$ bounds a disc around the puncture that contains the punctured discs bounded by $l_{2}$ and $l_{3}$. An analysis of the basepoint segment pattern shows that only case $A$ with pattern $\left(1,2,3,3^{\prime}, 2^{\prime}, 1^{\prime}\right)$ or case $B$ with pattern $\left(1,3,2,2^{\prime}, 3^{\prime}, 1^{\prime}\right)$ from Figure 2 are possible. In each of these cases, $w_{1}=w_{2}=w_{3}$ and

$$
[w]=\left[w_{1}^{3}\right]=\left[g\left(\left(a b a^{-1} b^{-1}\right)^{3}\right)\right] .
$$

For Case 4, we make use of methods from [5]. First, note that we can induce an automorphism between the $w_{i}$ and words in $a$ and $b$. Typically, we use $g(a)=w_{j}$ and $g(b)=w_{k}$ for $j \neq k$, but occasionally we use more complicated automorphisms in order to simplify a word. In addition, by an automorphism $g$, we actually mean some automorphism $g$. For example, $[g(a)]$, refers to any generator in $\pi_{1}(T)$.

The second method we adopt is the technique of cutting $T$ along some loop $l$ whose image in $\pi_{1}(T)$ is a generator to obtain a disk bounded by $l$ containing the puncture and a hole also bounded by $l$. We draw the resulting disc as in Figure 4, with the loop $l$ oriented clockwise around the outer boundary. Whenever possible, we choose the loop $l$ that we cut $T$ open along in such a way that the other loops have their base point at the left side of resulting figure as in Figure 4. Finally, we frequently introduce an additional path whose image in $\pi_{1}(T)$ forms a basis with the homotopy class of the loop bounding this disk.

Case 4: Two of $\left[w_{1}\right],\left[w_{2}\right],\left[w_{3}\right]$ are of the form $[g(a)]$ for some automorphism $g$ of $\pi_{1}(T)$, and the third is in $\left\{\left[a b a^{-1} b^{-1}\right],\left[\left[b a b^{-1} a^{-1}\right]\right\}\right.$.

Three subcases arise. The first is that the two homotopy classes that represent generators form a generating pair for $\pi_{1}(T)$. The second is that the two homotopy classes that represent generators do not form a generating pair for $\pi_{1}(T)$, and that these two homotopy classes are either equal or are inverses of each other. The third
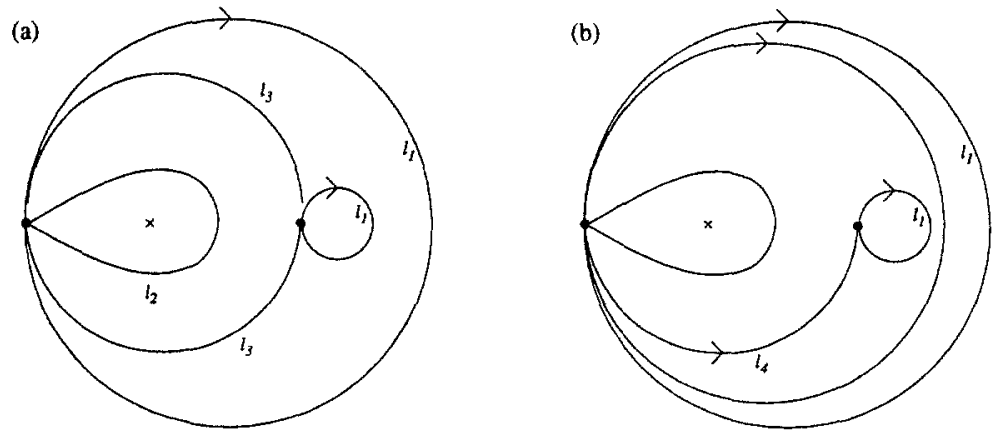

Figure 4. The torus $T$ cut along $l_{1}$ in the first and second parts of Case 4 . 
is that the two homotopy classes that represent generators do not form a generating pair for $\pi_{1}(T)$, and that these two homotopy classes are neither equal to each other nor are inverses of each other. We provide details only for the second subcase.

Second Subcase: Assume that the two homotopy classes that represent generators do not form a generating pair for $\pi_{1}(T)$, and that these two homotopy classes are either equal or are inverses of each other. Without loss of generality, $l_{1}$ is one of the homotopy classes representing a generator and the torus may be cut along $l_{1}$ to obtain a figure with the other loops positioned as in Figure 4 (b). Define a path $l_{4}$, disjoint except for the base point from $l_{1}, l_{2}$ and $l_{3}$ such that $w_{1}$ and $w_{4}$ form a generating pair for $\pi_{1}(T)$. An analysis of the basepoint segment pattern shows that only case $A$ with pattern $\left(1,2,3,3^{\prime}, 2^{\prime}, 1^{\prime}\right)$ or case B with pattern $(1,3$, $\left.2,2^{\prime}, 3^{\prime}, 1^{\prime}\right)$ are possible. So the other loop that represents a generator is homotopic to $l_{1}$ as indicated in the figure. In the first case $w_{2}=w_{1}$ and $w_{3}=w_{1} w_{4} w_{1}^{-1} w_{4}^{-1}$ and in the second case, $w_{3}=w_{1}$ and $w_{3}=w_{1} w_{4} w_{1}^{-1} w_{4}^{-1}$.

Thus,

$$
[w]=\left[w_{1} w_{2} w_{3}\right]=\left[w_{1} w_{1} w_{1} w_{4} w_{1}^{-1} w_{4}^{-1}\right]=\left[g\left(a a a b a^{-1} b^{-1}\right)\right],
$$

or

$$
[w]=\left[w_{1} w_{2} w_{3}\right]=\left[w_{1} w_{1} w_{4} w_{1}^{-1} w_{4}^{-1} w_{1}\right]=\left[g\left(a a b a^{-1} b^{-1} a\right)\right] .
$$

A conjugation shows that the above two classes are equal.

A similar analysis of the first subcase gives

$$
[w]=\left[w_{1} w_{2} w_{3}\right\}=\left\{g\left(a b^{-1} a b a^{-1} b\right)\right\}
$$

Similarly, in the third subcase one finds that either

$$
[w]=\left[w_{1} w_{2} w_{3}\right]=\left[g\left(a b a b^{-1} a b a^{-1} b^{-1}\right)\right],
$$

or

$$
[w]=\left[w_{1} w_{2} w_{3}\right]=\left[g\left(a b a^{-1} a^{-1} b^{-1} a\right)\right] .
$$

Case 3: A similar analysis yields

$$
[w]=\left[w_{1} w_{2} w_{3}\right]=\left[g\left(a a b a^{-1} b^{-1} a b a^{-1} b^{-1}\right)\right] .
$$

Case 5: One finds

$$
\begin{gathered}
{[w]=\left[w_{1} w_{2} w_{3}\right]=[g(a)],} \\
{[w]=\left[w_{1} w_{2} w_{3}\right]=\left[g\left(a^{3}\right)\right],} \\
{[w]=\left[w_{1} w_{2} w_{3}\right]=\left[g\left(b a b^{-1} a a\right)\right],} \\
{[w]=\left[w_{1} w_{2} w_{3}\right]=\left[g\left(a^{2}\right)\right],}
\end{gathered}
$$

or

$$
[w]=\left[w_{1} w_{2} w_{3}\right]=\left[g\left(a b^{-1} a b b\right)\right]
$$


We have completed the classification of the compositions of three simple loops that arise from loops with two transverse intersections. It remains to show that the classes that we obtained are distinct. This is done after the next two sections on the discussion of topological and geometric applications of this result. We now state the following result on curves with two nontrivial intersections.

Theorem 3.5. The conjugacy class in $\pi_{1}(T)$ of a loop on $T$ with two non-trivial self-intersections is one of

(a) $\left[g\left(a a b a^{-1} b^{-1} a b a^{-1} b^{-1}\right)\right]$,

(b) $\left[g\left(a a a b a^{-1} b^{-1}\right)\right]$,

(c) $[g(a a a b b)]$,

(d) $\left[g\left(a b a b^{-1} a b a^{-1} b^{-1}\right)\right]$,

(e) $\left[g\left(a a b a^{-1} a^{-1} b^{-1}\right)\right]$,

(f) $\left[g\left(a a b a b^{-1}\right)\right]$,

(g) $\left[g\left(a^{3}\right)\right]$, or

(h) $\left[g\left(\left(a b a^{-1} b^{-1}\right)^{3}\right)\right]$

for some $g \in$ Aut $\pi_{1}(T)$. Moreover, these classes are distinct.

Proof. We apply Theorem 3.4 and eliminate the simple loops, and loops with a single non-trivial self-intersection.

The only candidates remaining are (a)-(h) above. It is sufficient to demonstrate that each of these classes does not contain a simple loop or a loop with a single non-trivial intersection. Since each of classes (c) through (h) is primitive, specialization to the $\Gamma^{\prime}$ metric shows that two is the minimal self intersection number for each of these classes. Classes (a) and (b) are the non-primitive classes and a simple algebraic argument along with Theorems 1.1 and 1.2 shows that they do not contain simple loops or loops with a single non-trivial intersection. As mentioned above, the proof of distinctness is postponed until after a discussion of the geometric and topological consequences of the classification.

\section{Topological Consequences of the Classification}

Theorem 4.1. Each of the classes in Theorem 3.5 contains, up to homeomorphism, a unique curve of self intersection number 2. That is, if $l$ and $l^{\prime}$ are closed curves of self intersection number two in one of the classes from Theorem 3.5, then there is a homeomorphism $h$ of $T$ and an orientation preserving homeomorphism $k$ of $S^{1}$ so that $h \circ l=l^{\prime} \circ k$.

Proof. To obtain this result, it suffices to reexamine the proof of Theorem 3.4 and to recover the original curves with two self intersections by reversing the collapsing as described in Corollary 3.2. During the analysis carried out in the proof of Theorem 3.4, cyclically permuting the three curves making up the loop corresponds to applying an orientation preserving homeomorphism of $S^{1}$ prior to mapping $S^{1}$ into the punctured torus. The classes in Theorem 3.4 arise in a number of different cases during the analysis. In every instance, the different cases corresponding to the same class yield the same topological type of curve on reversing the collapsing process. The curves obtained are those in Figures 5 through 12 with one of the two possible orientations. 


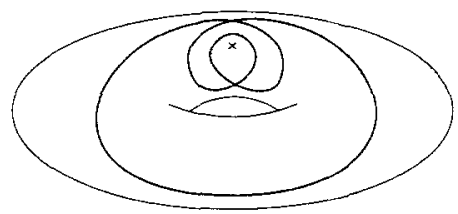

Figure 5. A loop on $T$ in the free homotopy class $\left[a a b a^{-1} b^{-1} a b a^{-1} b^{-1}\right]$.

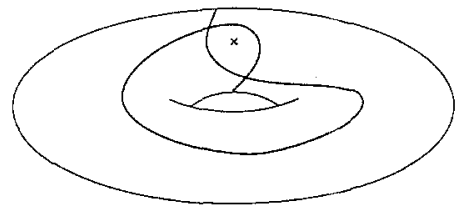

Figure 7. A loop on $T$ in the free homotopy class $[a a a b b]$.

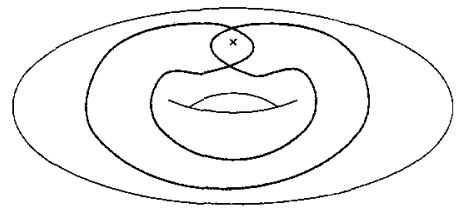

Figure 9. A loop on $T$ in the free homotopy class $\left[a a b a^{-1} a^{-1} b^{-1}\right]$.

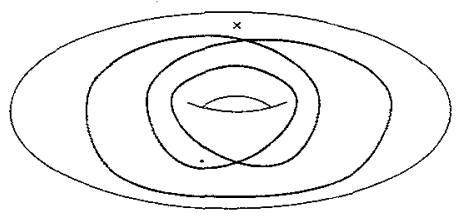

Figure 11. A loop on $T$ in the free homotopy class $\left[a^{3}\right]$.

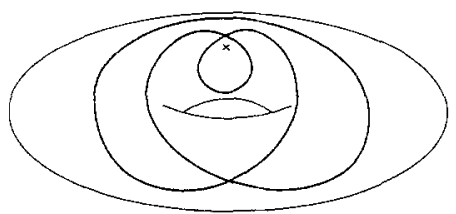

Figure 6. A loop on $T$ in the free homotopy class $\left[a a a b a^{-1} b^{-1}\right]$.

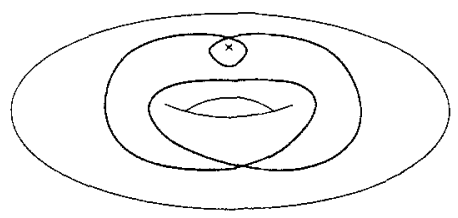

Figure 8. A loop on $T$ in the free homotopy class $\left[a b a b^{-1} a b a^{-1} b^{-1}\right]$.

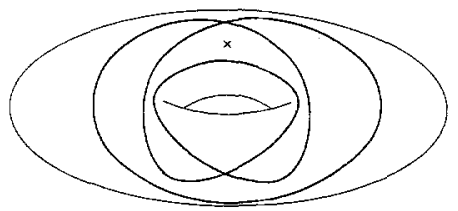

Figure 10. A loop on $T$ in the free homotopy class $\left[a a b a b^{-1}\right]$.

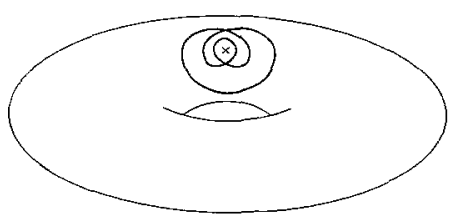

Figure 12. A loop on $T$ in the free homotopy class $\left[\left(a b a^{-1} b^{-1}\right)^{3}\right]$.

Note that for each of the figures, there is a self homeomorphism of the punctured torus that takes the given curve to itself with orientation reversed. The result now follows.

Note that in every diagram but the third, there are two possible segments to collapse to obtain a wedge of three simple loops as in Lemma 3.1. In the third case, there are four possible segments to collapse. This leads to this case occurring in apparently different subcases of Theorem 3.4. 
Theorem 4.2. Let $l$ be a closed curve of self intersection number 2 on the punctured torus $T$. Assume that $l$ is not freely homotopic to a curve with fewer self intersections. Then for all but two of the free homotopy classes listed in Theorem 3.5 , the class of $l$ is completely determined by closures of the complementary domains of the image of $l$ in T. For the remaining two classes, the class of $l$ is determined by the pattern of intersections of the closures of the complementary domains of the image of $l$.

Proof. It suffices to list the components and closures of components and to note the pattern of intersections. This is done in Table 1 below.

Note that all the classes above with the exception of the fourth and sixth are distinguished by the closures of the components of the complements of the curves. The fourth and sixth class are distinguished as follows. In the fourth class, the intersection of the closures of the first and second listed components is a single point. In the sixth class, the same intersection is the entire boundary of the closed punctured disc.

In the following table, $c$ represents $a b a^{-1} b^{-1}$.

Table 1. Topological types of twice self intersecting loops

\begin{tabular}{|c|c|c|c|}
\hline Class & $\begin{array}{l}\text { Number of } \\
\text { Components }\end{array}$ & Components & $\begin{array}{l}\text { Closure of } \\
\text { Components }\end{array}$ \\
\hline$\overline{a^{3}}$ & 3 & $\begin{array}{l}\text { punctured open cylinder } \\
\text { open disc } \\
\text { open disc }\end{array}$ & $\begin{array}{l}\text { closed cylinder } \\
\text { pinched cylinder } \\
\text { pinched cylinder }\end{array}$ \\
\hline$c^{3}$ & 4 & $\begin{array}{l}\text { punctured open disc } \\
\text { open disc } \\
\text { open disc } \\
\text { punctured open cylinder }\end{array}$ & $\begin{array}{l}\text { punctured closed disc } \\
\text { pinched cylinder } \\
\text { pinched cylinder } \\
\text { closed one handle }\end{array}$ \\
\hline$a c^{2}$ & 3 & $\begin{array}{l}\text { punctured open disc } \\
\text { open disc } \\
\text { open cylinder }\end{array}$ & $\begin{array}{l}\text { punctured closed disc } \\
\text { pinched cylinder } \\
\text { closed one handle }\end{array}$ \\
\hline$a^{2} c$ & 3 & $\begin{array}{l}\text { punctured open disc } \\
\text { open disc } \\
\text { open cylinder }\end{array}$ & $\begin{array}{l}\text { punctured closed disc } \\
\text { pinched cylinder } \\
\text { closed pinched one handle }\end{array}$ \\
\hline$a^{3} b^{2}$ & 2 & $\begin{array}{l}\text { punctured open disc } \\
\text { open disc }\end{array}$ & $\begin{array}{l}\text { punctured closed disc } \\
\text { closed one handle }\end{array}$ \\
\hline$a b a b^{-1} c$ & 3 & $\begin{array}{l}\text { punctured open disc } \\
\text { open disc } \\
\text { open cylinder }\end{array}$ & $\begin{array}{l}\text { punctured closed disc } \\
\text { pinched cylinder } \\
\text { closed pinched one handle }\end{array}$ \\
\hline$a a b a^{-2} b^{-1}$ & 3 & $\begin{array}{l}\text { punctured open disc } \\
\text { open disc } \\
\text { open cylinder }\end{array}$ & $\begin{array}{l}\text { punctured closed disc } \\
\text { punctured closed cylinder } \\
\text { closed cylinder }\end{array}$ \\
\hline$a a b a b^{-1}$ & 3 & $\begin{array}{l}\text { punctured open disc } \\
\text { open disc } \\
\text { open cylinder }\end{array}$ & $\begin{array}{l}\text { punctured pinched cylinder } \\
\text { pinched cylinder } \\
\text { closed cylinder }\end{array}$ \\
\hline
\end{tabular}




\section{Geometric Consequences of the Classification}

We identify those classes which contain geodesics with two self-intersections. This proof parallels Theorem 3.2 in [5], but is included for completeness.

Theorem 5.1. A closed geodesic on Thas two self-intersections if and only if it is in one of the following free homotopy classes

(a) $\left[g\left(a a b a^{-1} b^{-1} a b a^{-1} b^{-1}\right)\right]$,

(b) $\left[g\left(a a a b^{-1} b^{-1}\right)\right]$,

(c) $[g(a a a b b)]$,

(d) $\left[g\left(a b a b^{-1} a b a^{-1} b^{-1}\right)\right]$,

(e) $\left[g\left(a a b a^{-1} a^{-1} b^{-1}\right)\right]$, or

(f) $\left[g\left(a a b a b^{-1}\right)\right]$

for some $g \in$ Aut $(F(a, b))$. Any two geodesics in the same class are of the same topological type. Moreover, these free homotopy classes are distinct.

Proof. Closed geodesics on $T$ lie in primitive free homotopy classes and realize the minimum number of self-intersections for their free homotopy classes. We apply Theorem 3.5 and exclude the non-primitive classes $\left[g\left(\left(a b a^{-1} b^{-1}\right)^{3}\right)\right.$ and $\left[g\left(a^{3}\right)\right]$ Since the remaining classes are primitive, each class contains a geodesic. By the topological classification in the preceding section, there is only one topological type of geodesic in each class.

It remains to be shown that these classes are distinct. This follows from the result in the next section.

\section{Distinctness of the Classes}

Theorem 6.1. The classes obtained in Theorem 3.5 are all distinct.

Proof. We indicate several means of proof. First, note that $\left[g\left(a^{3}\right)\right]$ and $\left[g\left(a b a^{-1} b^{-1}\right)^{3}\right]$, being imprimitive, are distinct from the other classes. The aforementioned result of Nielsen shows that they are also distinct from each other.

Approach A. An algorithm for deciding whether there is an automorphism of $F(a, b)$ taking a specific primitive word $w_{1}$ to a specific primitive word $w_{2}$ has been described by J.H.C. WHITEHEAD [17]. One can apply this in our situation and prove the Theorem.

Approach $B$. We now use the fact that every automorphism of a punctured torus can be realized by a diffeomorphism which preserves the set of geodesics under a given metric. The background for this was briefly summarized in Section 2.2; the main implication is that two primitive free homotopy classes can be in the same homeomorphism class only if the topological type of the associated geodesics for any fixed hyperbolic metric are the same. In particular, we can specialize to the $\Gamma^{\prime}$-metric and find that the information of Table 1 guarantees that the Theorem is true. 
Approach $C$. Note that two loops on $T$ cannot be in automorphic conjugacy classes in $\pi_{1}(T)$ if they are not in automorphic conjugacy classes on the usual, non-punctured, torus $T^{\prime}$. When we remove the puncture from $T$, loops can be deformed as follows:

(i) $\left[a a b a^{-1} a^{-1} b^{-1}\right]$ is in $[\mathrm{Id}]^{\prime}$

(ii) $\left[a a b a^{-1} b^{-1} a b a^{-1} b^{-1}\right]$ and $[a a a b b]$ are in $[g(a)]^{\prime}$

(iii) $\left[a a a b a^{-1} b^{-1}\right]$ and $\left[a b a b^{-1} a b a^{-1} b^{-1}\right]$ are in $\left[g\left(a^{2}\right)\right]^{\prime}$

(iv) $\left[a a b a b^{-1}\right]$ is in $\left[g\left(a^{3}\right)\right]^{\prime}$

for some $g \in$ Aut $\pi_{1}(T)$. This enables us to distinguish all but two pairs of classes.

We show directly that there are no automorphisms taking $a C C$ to $a a a b b$, nor $a a C$ to $a b a b^{-1} C$, where $C:=a b a^{-1} b^{-1}$. To this end, we recall two results of $\mathbf{J}$. NIELSEN [11]: (1) a cyclically reduced word $W$ in $a$ and $b$ is a generator of $F(a, b)$ only if all exponents of $a$ in $W$ are of the same sign, similarly for those of $b$; (2) automorphisms preserve the set of conjugates of $C^{ \pm 1}$.

If in the first of these cases there were such an automorphism $\phi$, then we could solve for $\phi(a)=a a a b b V$, with $V=X C^{ \pm 2} X^{-1}$. This right hand side cannot reduce to have either of $a$ or $b$ appear to the same exponent sign throughout. Since an automorphic image of $a$ is a generator, this right hand side must not be cyclically reduced. But this implies that there is an automorphism $\psi$ such that $\psi(a)=a a b b a Y C^{ \pm 2} Y^{-1}$. Again, this cannot be reduced so as to have same sign exponents of $a$ nor $b$. Indeed, proceeding cyclically, we find that there can be no such $\phi$.

In the second case, an initial automorphism allows us to consider the existence of a $\phi$ with $\phi\left(a^{2}\right)=a b b a C W$, with $W=X C^{ \pm 1} X^{-1}$. But, if $W$ is not $C^{-1}$, there can be no reduction so as to produce a right hand side of same sign exponents in either $a$ or $b$. Hence, either $\phi\left(a^{2}\right)=a b b a$, or this right hand side is not cyclically reduced. Proceeding through all possible cyclic permutations, we find that $\phi\left(a^{2}\right)$ must be equal to one of the cyclic permutations of $a b b a$.

Now, if some $Z=w_{1} U w_{2}$ for letters $w_{i}$ and word $U$, then the reduced word of $Z^{2}$ is either $w_{1} U w_{2} w_{1} U w_{2}$ or $w_{1} U^{2} w_{2}$. This latter case arises only if $w_{1}=w_{2}^{-1}$. It is easily checked that for the above choices, $\phi\left(a^{2}\right)$ cannot have the form of a square. Hence, no such automorphism exists.

\section{Applications to the Markoff Spectrum}

Recall from the discussion in Section 2.3 that A. Haas has shown that a closed geodesic for the $\Gamma^{\prime}$-metric corresponds to a Markoff value greater than 6 if and only if the geodesic contains a loop about the puncture. We thus conclude the following.

Lemma 7.1. Of all twice self-intersecting geodesics on $\Gamma^{\prime} \backslash \mathscr{H}$, only those which are automorphic images of (i) $A^{3} B^{2}$, (ii) $A^{2} B A B^{-1}$ or (iii) $A^{2} B A^{-2} B^{-1}$ have Markoff values less than 6.

Our new examples of geodesics has led us to the following

Theorem 7.2. Let $W(A, B)$ be a cyclically reduced primitive word in $A$ and $B$ of positive exponents. Then the Markoff values of the images of $W$ under the 
automorphisms of $F(A, B)$ lie between $\sqrt{5}$ and 6 . Furthermore, 3 is a limit value of this set of Markoff values.

Proof. Since $W(A, B)$ is a primitive word in $A$ and $B$ of positive exponents, it clearly has no occurrence of the commutator of $A$ and $B$ within it. In particular, the corresponding geodesic does not contain a loop about the puncture. Therefore neither do any of the geodesics corresponding to automorphic images of $W$. But, by the remark of Haas, this implies that the corresponding Markoff values are then at most 6. It is well known that the minimum Markoff value is $\sqrt{5}$.

Let $C=B^{-1} A^{-1} B^{-1}$ and $D=B^{-1}$. Then $(C, D)$ is a generating pair for $F(A, B)$. In the $\Gamma^{\prime}$-metric, $C$ acts as $C z=[2 ; 2, z]$ and $D$ as $D z=[1 ; 1, z]$. Thus words in positive powers of $C$ and $D$ correspond to (doubly infinite) sequences whose entries are double $1 \mathrm{~s}$ and double $2 \mathrm{~s}$.

The work of [5], [6] and [7] shows that there is a binary tree of isometries, so called Dehn twists, generated by $\lambda$ and $\rho$; branching to the left is given by $\lambda$ which replaces $C$ by $C D$ and fixes $D$, while branching to the right is given by $\rho$ which replaces $D$ by $C D$ and fixes $C$. Now, since the automorphism group of $\Gamma^{\prime}$ is generated by $\lambda$ and $\rho$, the corresponding tree of images of $W(C, D)$ is indeed infinite.

Each of these images of $W$ has a corresponding periodic sequence of double $1 \mathrm{~s}$ and double 2s; furthermore, as one penetrates deep into the tree, the sequences have ever longer subsets which agree with the periods corresponding to the simple geodesics. Thus, the images of $W$ correspond to Markoff values approaching the limit value of the Markoff values of the simple geodesics. But, this unique limit value is well known to be 3 .

Corollary 7.3. The automorphism classes of (i) $A^{3} B^{2}$ and (ii) $A^{2} B A B^{-1}$ have Markoff values which admit 3 as a limit.

Proof. That $A^{3} B^{2}$ meets the hypotheses of the Theorem is obvious. As to $A^{2} B A B^{-1}$, we note that the automorphism given by $(A, B) \rightarrow(A B, B)$ converts this to a word in positive powers of $A$ and $B$.

We now address the third class of "low height" twice self-intersecting geodesics.

Proposition 7.4. The automorphic images of $A^{2} B A^{-2} B^{-1}$ have Markoff values between 3 and 6. Furthermore, 6 is the unique limit value of this set of Markoff values.

For typographical reasons, in what follows, we use $\bar{X}$ to denote the inverse of a group element $X$.

Proof. The automorphism $(A, B) \rightarrow(D C, \bar{D})$, sends $A^{2} B \bar{A}^{2} \bar{B}$ to $(D C)^{2}(\bar{D} \bar{C})^{2}$. We again restrict to the tree of images corresponding to repeated applications of the automorphisms $\lambda$ and $\rho$ as above. Let $\alpha$ be one of the resulting composite automorphisms. That is, suppose $\alpha=x_{1} \cdots x_{n}$ where each $x_{i}$ is $\lambda$ or $\rho$.

By cyclically permuting, we replace $(D C)^{2}(\bar{D} \bar{C})^{2}$ by $C \bar{D} \bar{C} \bar{D} \bar{C} D C D$. We will consider the effect of applying $\alpha$. Using induction, it is not hard to verify that 
$\alpha(C D)=C W D$ where $W$ is a symmetrical word in positive powers of $C$ and $D$. Therefore, $(\alpha(C D))^{-1}=\bar{D} \bar{W} \bar{C}$.

Claim. For all $\alpha$ as above, $\alpha(C \bar{D} \bar{C} \bar{D} \bar{C} D C D)=C \bar{D} \cdot \bar{C} \bar{W} \bar{D} \cdot \bar{C} D \cdot C W D$.

The proof is by induction on $n$, the "length" of $\alpha$. It thus suffices to check applications of $\lambda$ and $\rho$ to words of the form indicated. Applying $\lambda$, we have

$$
\begin{aligned}
\lambda \alpha(C \bar{D} \bar{C} \bar{D} \bar{C} D C D) & =\lambda(C \bar{D} \cdot \bar{C} \bar{W} \bar{D} \cdot \bar{C} D C W D) \\
& =C \bar{D} \cdot \bar{C} \lambda(\bar{W}) \bar{D} \bar{D} \cdot \bar{C} D \cdot C D \lambda(W) D .
\end{aligned}
$$

Since $\lambda \alpha(C D)=\lambda(C W D)=C D \lambda(W) D$, we find $W_{\lambda \alpha}=D \lambda(W)$ and we are done in this case. Similarly, applying $\rho$, we have

$$
\begin{aligned}
\rho \alpha(C \bar{D} \bar{C} \bar{D} \bar{C} D C D) & =\rho(C \bar{D} \cdot \bar{C} \bar{W} \bar{D} \cdot \bar{C} D \cdot \bar{C} W D) \\
& =C \bar{D} \cdot \bar{C}^{2} \rho(\bar{W} \bar{D} \cdot \bar{C} D \cdot C \rho(W) C D .
\end{aligned}
$$

But, $W_{\rho \alpha}=\rho(W) C$ and our claim follows.

We can now describe the transformation $\alpha(C \bar{D} \bar{C} \bar{D} \bar{C} D C D)$ as a continued fraction. We have $\alpha(C \bar{D} \bar{C} \bar{D} \bar{C} D C D)=C \bar{D} \cdot \bar{C} \bar{W} \bar{D} \cdot \bar{C} D \cdot C W D$. One easily checks that

$$
\begin{aligned}
C \bar{D} \bar{C}(z) & =[5 ; 2,-1 / z] ; \\
\bar{D}(z) & =-1 /([1 ; 1,-1 / z]) \\
\bar{C} D C(z) & =-1 /([5 ; 2, z]) ; \\
D(z) & =[1 ; 1, z] .
\end{aligned}
$$

Since $W$ is a word in positive powers of $C$ and $D$, we also have

$$
W(z)=\left[a_{0} ; a_{1}, \ldots, a_{n}, z\right]
$$

where $a_{0} ; a_{1}, \ldots, a_{n}$ is a sequence of 1,1 's and 2,2's. It follows that

$$
\bar{W}(z)=-1 /\left(\left[a_{n}, a_{n-1}, \ldots, a_{0},-1 / z\right]\right) .
$$

Using the symmetry of $W$ we therefore have $\bar{W}(z)=-1 /\left(\left[a_{n}, a_{n-1}, \ldots, a_{0}\right.\right.$, $-1 / z]$ ).

We now compose the various continued fractions to conclude that

$$
\begin{aligned}
\alpha\left(C(\bar{D} \bar{C})^{2} D C D\right)(z) & =C \bar{D} \cdot \bar{C} \bar{W} \bar{D} \cdot \bar{C} D \cdot C W D(z) \\
& =\left[5 ; 2, a_{0}, a_{1}, \ldots, a_{n}, 1,1,5,2, a_{0}, a_{1}, \ldots, a_{n}, 1,1, z\right] .
\end{aligned}
$$

The associated doubly infinite sequence of integers is $\mathscr{A}=(5,2$, $\left.a_{0}, a_{1}, \ldots, a_{n}, 1,1\right)^{\infty}$. Now recall that $\alpha(C D)=C W D$ and that this word corresponds to a simple closed geodesic, whose Markoff value hence is a Markoff number - one of a discrete set of values lying between $\sqrt{5}$ and 3 . Of course, $\alpha(C D)(z)=C W D(z)=\left[2 ; 2, a_{0}, a_{1}, \ldots, a_{n}, 1,1, z\right]$, of associated sequence $\mathscr{B}=\left(2,2, a_{0}, a_{1}, \ldots, a_{n}, 1,1\right)^{\infty}$. It follows that the Markoff value of $\mathscr{A}$ is exactly the Markoff value of $\mathscr{B}$ plus 3 . 
Remark. It is likely that each of the above two classes which admit 3 as a limit of their Markoff values actually admit this as their unique limit. However, we have included only the above proof of this nature.

We now discuss a property of possible arithmetic interest which differentiates the two classes which admit 3 as a limit of their Markoff values. M. Sheingorn and one of the authors has noted that many of the families of isolated values in the Markoff spectrum correspond to geodesics which enjoy a special symmetry. Briefly, A. Schmidt pointed out that every hyperbolic punctured torus admits a unique Riemann surface which it double covers. The covered surface is a punctured sphere with 3 points of ramification, each of order two. (Indeed, there is an elliptic involution on the torus; dividing by the involution, we find the sphere. The expected fourth point of ramification corresponds to the puncture itself.) In the case of $\Gamma^{\prime}$, this punctured sphere is $\Gamma^{3} \backslash \mathscr{H}$, where $\Gamma^{3}$ is the normal subgroup generated by all of the cubes in the full modular group. We call a closed geodesic of this surface a bouncer if it passes through two of the elliptic fixed points of order two. SHEINGORN [16] showed that the simple closed geodesics of $\Gamma^{\prime} \backslash \mathscr{H}$ project to be bouncers; [14] shows that the proper singly self-intersecting geodesics of [6] do as well; in fact, various infinite families of known isolated values correspond to bouncers [14].

Of the twice self-intersecting geodesics, we now show that only those which are images of $A^{3} B^{2}$ are bouncers. It is known that $\Gamma^{3}$ is generated by the three elliptic elements, each of order two, with no non-trivial relations amongst them:

$$
T_{0}=\left(\begin{array}{rr}
0 & -1 \\
1 & 0
\end{array}\right), \quad T_{1}=\left(\begin{array}{ll}
1 & -2 \\
1 & -1
\end{array}\right), \quad T_{2}=\left(\begin{array}{ll}
2 & -5 \\
1 & -2
\end{array}\right) .
$$

It is easily checked that $A=T_{1} T_{2} T_{1} T_{0}$ and $B=T_{0} T_{1}$. Note that since each $T_{i}$ is its own inverse, each of $A$ and $B$ is the product of two elliptic elements (elements of trace 0 ) in $\Gamma^{3}$. This shows that the corresponding geodesics are indeed bouncers. It is fairly straightforward to check that the automorphisms of $\Gamma^{\prime}$ preserve the set of elements which are products of two elliptics of $\Gamma^{3}$. Thus, we can now write out words for each of cases (c), (e) and (f) in terms of $A$ and $B$, substitute to obtain words in the $T_{i}$ and check. (There are perhaps more elegant ways to perform this check, but the present manner is probably the most accessible.) For an explicit member of this type, we have

$$
A^{3} B^{2}=T_{1}\left(T_{2} T_{1} T_{0} T_{1}\right) T_{2}\left(T_{1} T_{0} T_{1} T_{2}\right) T_{0} T_{1} .
$$

Thus, by conjugating by $T_{1}$, we see that the corresponding geodesic bounces from the projection of the elliptic fixed point of $T_{2}$ to that of $T_{0}$.

One easily checks the other classes, so as to conclude the

Proposition 7.5. Of the twice self-intersecting geodesics on $\Gamma^{\prime} \backslash \mathscr{H}$, only those which are automorphic images of $A^{3} B^{2}$ project to geodesics of $\Gamma^{3} \backslash \mathscr{H}$ which bounce between two elliptic fixed points of order two.

Whereas [14] shows that each proper singly self-intersecting geodesic of [6] is forced to have its point of intersection at one of the three pre-images of the points 
of ramification, it is not the case that these bouncing 2-self-intersectors have these as their points of self-intersection. A quick calculation readily shows this for the geodesic of $A^{3} B^{2}$.

Remark. We note that the results of even this section are quite strongly independent of hyperbolic metric. A. HaAs [9] showed that for any such metric, the simple closed geodesics have a particular "height" as their limit. Similarly, for a fixed hyperbolic metric, the property of a geodesic having a loop about the puncture can be expressed in terms of a fixed height (basically, this is given by the translation width at infinity of the corresponding group). Furthermore, the work of M. SHEINGORN [16] indicates that a word corresponding to a geodesic with respect to a fixed hyperbolic metric on a punctured torus which projects to a bouncing geodesic on the punctured sphere double covered by the torus will have this same property with respect to any hyperbolic metric.

\section{Final Comments}

The techniques described in this paper can be used to obtain a classification of curves with higher self intersection number than two on the punctured torus. The analysis becomes more complicated because the number of cases increases.

It would be interesting to see if it is always the case that for a fixed $n$, the free homotopy classes of self-intersection number $n$ are distinguished by their topological types (including complements).

Our results on the Markoff spectrum also lead to various questions. It would be quite interesting were it the case that one of the automorphism classes would give isolated Markoff values, as conjectured by Crisp and Moran for the proper single self intersectors.

\section{References}

[1] BiRman J, Series C (1984) An algorithm for simple curves on surfaces. J London Math Soc (2) 29: $331-342$

[2] BUSER P (1992) Geometry and Spectra of Compact Riemann Surfaces. Boston: Birkhäuser

[3] Buser P, SEMmler K-D (1988) The geometry and spectrum of the one holed torus. Comment Math Helv 63: $259-274$

[4] Corn H (1993) Markoff geodesics in matrix theory. In: Pollington A, Moran W (eds) Number Theory with an Emphasis on the Markoff Spectrum, pp 69-82. New York: Dekker

[5] CRISP D (1993) The Markoff Spectrum and Geodesics on the Punctured Torus. PhD Thesis Univ of Adelaide

[6] CrISP D, Moran W (1993) Single self-intersection geodesics and the Markoff spectrum. In: Pollington A, Moran W (eds) The Markoff Spectrum, Diophantine Analysis and Analytic Number Theory, pp. 83-94. New York: Dekker

[7] Crisp D, Moran W (1995) The Markoff spectrum and geodesics with one self-intersection on the punctured torus. Flinders Univ Preprint

[8] Cusick TW, Flahive ME (1989) The Markoff and Lagrange Spectra. Providence, RI: Amer Math Soc

[9] HAAS A (1988) Diophantine approximation on hyperbolic orbifolds. Duke Math J 56: 531-547

[10] Maskit B (1989) Parameters for Fuchsian groups II: topological type (1, 1). Ann Acad Sci Fennicae 14: 365-375

[11] NreLSEN J (1918) Die Isomorphismen der allgemeinen unendlichen Gruppe mit zwei Erzeugenden. Math Ann 78: 385-397

[12] Rolfsen D (1990) Knots and Links. Houston, Texas: Publish or Perish 
[13] SсHмIDT A (1976) The minimum of quadratic forms with respect to Fuchsian groups, I. J Reine Angew Math (Crelle) 287: 341-368

[14] SCHMIDT TA, SHEINGoRN M (1997) Markoff geometry on $\Gamma^{3} \backslash \mathscr{H}$. Oregon State Univ Preprint

[15] SERIES C (1985) The geometry of Markoff numbers. Math Intel 7(3): 20-29

[16] SHEIngorn M (1985) Characterization of simple closed geodesics on Fricke surfaces. Duke Math J 52: 535-545

[17] WHTTEHEAD JHC (1936) On equivalent sets of elements in a free group. Ann Math 37: 782-800

[18] WolPERT S (1983) On the Kähler form of the moduli space of once punctured tori. Comment Math Helv 58: $246-256$

[19] ZIESCHANG H (1986) Minimal geodesics of a torus with a hole (Russian). Izv Acad Sci USSR 50: 1097-1105; English transl. in: Math USSR Izv 29: 449-457 (1987)

D. CRISP

Flinders University

Department of Mathematics

Adelaide 5001

Australia

\section{S. Dziadosz}

University of Michigan

Department of Mathematics

Ann Arbor, Michigan 48109

USA

D. J. GARITY

Oregon State University

Department of Mathematics

Kidder Hall 368

Corvallis, Oregon 97331-4605

USA
T. INSEL

University of California Department of Mathematics Berkeley, California 94720

USA

T. A. SchMidT

Oregon State University Department of Mathematics

Kidder Hall 368

Corvallis, Oregon 97331-4605

USA

P. WILES

University of Wisconsin

Department of Mathematics

Madison, Wisconsin 53706

USA 